\title{
SINONOMA DE HERBIS
}

p. 245 Alleluya, panis cuculi; payn de cukulle; wodesowre, stubwort. Amantilla, potentilla, maturella, valeriana; valerian.

Allipiados, laureola, herba catholica; laureole.

Allium, scordion, tiria[c] a rusticorum; ail; id est garleek.

$5 \quad$ Arthemesia, matricaria, mater herbarum; hermoise; mugwort.

Althea, bismalua, euiscus, malua-viscus; wismalwe; merchmalwe, holihocke.

Arnoglossa, plantago maior; plantayne; weybrede.

Alphita, nomen ordei; fereine de orge; bere mele.

10 Auancia, gariophilata, pes leporis, sanamunda; harefoot, auence.

Adianthos, pollitricum, pes nisi; sparhaukfot.

Attriplex, crisolocanna; id est arrage.

Archangelica; pe blynde nettyl.

Agrimonia; egrimonie; garschelme.

15 Apium per bep v spices of hem: apiu $m$ domesticum, apium ranarum, apium ranis, apium emeroydarum.

Ache; merch, smalache.

Abrotanum; au[e]royne; southerenewode.

Anetum, absinthium dulce; di-le; ane [t].

20 Aloes per bep iij: aloes epatyk, aloe cicatrinum (pese ben put in medicines), aloes caballinum ys put in plastres. Aloes lignium comep out of Paradys and pat oper comep out of Inde.

Anagallus, anagallicum, consolida maior; and pe rote of hym ys clepid simphitum, comfyrie.

25 Aquilina, columbaria; columbyn.

Auricula lepor[is]; hals-wort, orpyn.

Aaron, barba Aaron, pes vituli; [pe de] polayn; zekesters.

Aquilaria, celidonya; celidonye; te[t] erwort.

Absynthium; aloyne; wormood.

30 Anthemis, camamilla; camamille.

Anisium, siminu $m$ dulce; anyes.

p. 246 Antimonium it is a veyne of pe erpe lich antymonye; | id est leederpe.

23 rote: symphitu[m] comfery added in the outer margin by a later hand.

4 tiriaca] tiriata MS. 18 aueroyne] auoroyne MS. 19 anet] aner MS.

26 leporis] lepora $M S .27$ pe de] pede MS. 28 teterwort] tederwort $M S$. 
And $[\mathbf{r}]$ ago, portulaca; parcelane.

Achorus it is pe rote of gladyole.

35 Anachardus it is pe lous of an olyfant and sum-tyme fruyt of a tre.

Alica, [spelta; spelt].

Aspaltum, butimen iudaicum.

Albeston, calx viua; viuez schauz; quiklym.

Anochorus, saniculus; sanicle; wodemerch.

40 Ambrosia; hindehale, wilde sawge; eupatorium; ambrosie; saluia agrestis.

Amidum; amydoyne, pe ious per-of is swete.

Ambra, sperma ceti; id est ambre.

Azarus, azarabaccara, gariophilata agrest $i$.

45 Ayson, barba Iouis, semper-viua; rubarbe, herwort, seluegrene.

Astula regia, herba mus[c]ata; woderoue.

Acetosa; surel; surdok.

Agradiana; id est hofe.

[Andraphasis]; id est melde.

50 Anochana, brionida, cicida; [wilde nep], [wilde vine].

Aspaltyos, quinque-folium, pe[n]tafilon; synk-foyl.

Aristologia; smerwort.

Acetosum, acetum; vynegre or eysel.

Arilli bep pe kernelys of re[y]sons.

55 Aattramentum it is a veyne of pe erthe, of whiche per bep tweyn: on ys blak, an-oper is grene, coprose, pe whiche is clepid vitriole. Acacia is pe ius of litle smale slon, pe which ben nozt fulleche rype.

Alyp[ia]dos; laureole, and pe sed ys clepid coconidium.

60 [Affodillus], centum capita.

Allogalica, genciana.

Antera; pe sed of roses.

Ariogoda; sumak.

Antale, id est aunctum dulce.

65 Arnoglossa, centineruia, li[n]gua arietis, plantago maior.

Antepholi; pe grete clowe j-fundyn among opere clowes.

Alexander; pe sed of hockont.

33 andrago] andago $M S . \quad 36$ spelta spelt] spleta splet $M S . \quad 46$ muscata] mustata MS. 49 andraphasis] archaprasis $M S . \quad 50$ wilde nep] wildenep MS. [ wilde vine] wildevine $M S . \quad 51$ pentafilon] petafilon $M S . \quad 54$ reysons] resons $M S . \quad 59$ alypiados] alypraidos $M S . \quad 60$ affodillus] affrodillus $M S$. 65 lingua] ligua $M S$. 
Absinthium amarum vel domesticum; wormod; al[o]yne.

Absinthium ponticum, centonica; wormod.

Acantum, oruum, ygida.

p. 247 A|galido, semen vrtice; pe sed of nettelys.

Acedula, herba acetosa, rume[x], oxilappacium; sorel; sourdokke.

Acinum; id est hyndeberye.

Actis, sambucus; helrene-tre.

75 Acus mus[c] ata maior, herbe roberd.

Acus mus[c] ata minor, $\mathrm{i} d$ est crowpile.

Agrimilla[t] $\mathbf{u m}$, gith, mellancium, ni-gella.

Acuialici, semen saxifrage.

Acride, pastinaca, ba[u]cia; pastir-nep.

80 Adianthos, alacon, capillus Veneris; mayden-her.

Algon, pollitricum; sparhawkfot.

Allium, tiriaca rusticorum; garlek.

$\mathbf{A}[\mathbf{n d r}] \mathbf{a g n i s}$, portulaca, albagla, pes pulli.

Alabim, cepa marina, cepa maior, cepa ca[n]ina; squylles.

85 Alapsa, galla, pomu $m$ quercinum.

Alga; sewor [wort]; coriaundre.

Alexandrum, olexatrum, petro[c]illum macedoni[c]um.

Alphita, farina ordei; flour of barlich.

Alphur; flour of ache.

$90 \quad$ Allephalesophei, lingua auis.

Albipha; pe appel of mandrake.

Alcami[r]a, fermentum.

Alumen, stiptera, zuccarium; id est alum de plume.

Alumen glaciei; alum de glas.

95 Amar[u]sca fetida vel [fetida cotula], mas[matr] um, peucedanum, $\mathrm{f}[\mathrm{e}]$ niculus porcinus; amerok, hondz-feue, mayn.

Amar[a]cus, amaricori, cimbrium, sansucus, olimbrium, maiorana; mugwort.

Amphoricon, ypericon, herba Johanis, herba perforata, scopa regia, fuga demonum; Johanys-wort.

Amy, fructus iuniperij.

68 aloyne] alayne $M S . \quad 72$ rumex] rumei $M S . \quad 75$ muscata] mustata $M S$. 76 muscata] mustata $M S .77$ agrimillatum] agrimillacum $M S .79$ baucia] bancia $M S .83$ andragnis] adriagnis $M S .84$ canina] camina $M S$. 86 wort] lbor MS. 87 petrocillum] petrotillum MS. | macedonicum] macedonium MS. 92 alcamira] alcamita $M S . \quad 95$ amarusca] amarisca $M S . \quad$ fetida cotula] fecula cotida MS. | masmatrum] masinatum MS. 96 feniculus] foniculus MS. 97 amaracus] amaricus MS. 
Antipharma[c] $\mathbf{u} m$, vicetoxicum; turmentille; and is good a-zens venym.

Anthos; pe flour of rose-maryn and ey wip-outyn schelle.

105 Aqua rosarum; enrose.

Bvglossa, lingua siluana, lingua bovis; [lange de bef].

Brascica, caulus; clous, choler; cool-wortes.

Bra[n]ca vrcina; bra[n]ch vrsine.

p. 248 Bra[t]heos; | sauina; saueyne.

110 Brionia, sicides; wildenep; cucurbita agrestis; brionye.

Bard[a]na, glis, lappa; clotere.

Bedegar, vulnis; eglenter; zelwbere.

B[i] rula; birle.

Bvr[i]th, s[a]ponaria; crowsope; card[uu]s fullonus; sauo[un]ere.

Balsamita; bawme.

Bruncus, cuscuta, [podagra lyni]; doder.

Bvtalmon, oculis bovis; oyle de be[u]f; herba paralisis; kousloppe.

Belliculi marini beth smale stones and white whiche beth fondyn be pe brynke of pe see.

120 Borage; borage.

Betonica; betoyne.

Benedicta herba; herbe benyt; homelok.

Bugla; bugle; wodeborne.

Bruscus, fragonum; [kn]ovholm, brusk.

Basilica, dragancia; cerpentina, dragaunce.

Bolus armoniacus; bole armonyak.

Ben rubium it is a rote pe whiche is y-foundyn on Hynde and pe white on Egypte.

Baucia, pastinaca; pastinake, pastre-nep.

Baccara, azara-baccara; spewyng-wort.

Balsam[i]ta, menta aquatica, ortensis, sizimbrium.

Barbastus, tapsus barbast $u s$, folsinus, ponfilyon.

Bacce lauri, fructus lauri, dampnococti et dampnocosti.

Baccarum edere, fructus edere, carpo[c]issi.

Barba ir[c]ina, ypoquistidos.

102 antipharmacum] antipharmatum MS. 106 lange de bef] lan gedebef $M S$. 108 branca] brauca MS. | branch] brauch MS. 109 bratheos ] bracheos MS. 111 bardana] bardona $M S .113$ birula] bvrula $M S .114$ bvrith] bvrth $M S$. saponaria] soponaria MS. | carduus] cardinis MS. | sauounere] sauonuere MS. 116 podagra lyni] padagralyni MS. 117 beuf] beif MS. 124 knovholm] buovholm MS. 131 balsamita] balsamunta MS. 134 carpocissi] carpotissi MS. 135 ircina] irtina $M S$. 
Bu-timen iudaicum, aspaltum.

Borax; pe gumme of a tre.

Bruscus; bonwort.

$\operatorname{Br}[\mathbf{u n}]$ cus, rasca lyni, podagra lyni, cusc $[u]$ ta.

140 Bursa pastoris, sanguinaria; stanche.

Consolida media; wryngyngwort; [meddel consoud].

Consolida minor; brusewort.

Ciclamen id est cassarium, panis porcinus, [nu]x terre, malum terre; noys de tere; erthe-note.

145 Camedreos, germandria; germandrie.

Colophonia, pix greca; colophonye.

p. 249 Cantabrum, fur|fur, tritici; bren of whete.

Capitellum, lexiuium; 1[e]ssyue; lizeus.

Ceru[s]a, album plumbum; blank plum; whit leed.

150 Caprifolium, mater silue; chiuerfoyl; wodebynde.

Caniculata, ius-quiami, simph[o]ni[a]ca, fistula; che[ni]le; hennebane.

Centaurea, fel terre; certorie; erthe[-gall].

Celidonia; me[mi] tha; celidonie de mere.

155 Crassula minor, vermicularis; stonore.

Crassula maior; orpyn.

Calamentum; calamy $n$ te.

Canabum; caun[v]re; henp.

Caballus marinus, ypotamus.

160 Centum gra $[\mathbf{n}] \mathbf{a}$, herba cancri.

Capud monarchi, dens leonis; dent de leon; lyonys toth.

Cruciata per bep ii of hem; herbe croyse.

Centinodium, li[n]gua passeris; swynesgres.

Cepe; onyouns.

165 Cepa marina, squilla; squillez.

Cima rubi; cy[uon]s de rounse; brimel-crop.

Capillis Veneris; mayden-her.

Cinamomum; kanel.

Calendula, solsequium, sponsa solis; solcicle; golden rodene.

139 bruncus] branucus MS. | cuscuta] cuscata MS. 141 meddel consoud] meddelconsond MS. 143 nux] mix MS. 148 lessyue] lossyue MS. 149 cerusa] cerula MS. 151 simphoniaca] simphanica MS. | chenile] chemle MS. 153 erthe-gall] erthe $M S .154$ memitha] menutha $M S .158$ caunvre] canure MS. 160 grana] graua MS. 163 lingua] ligua $M S .166$ cyuons] cinous MS. 
170 Consolida per bep iii of hem; comferie; wryngenge-wort; dayes-ezes.

Camamilla; camamylle

[Cymynum] dulce; a $[\mathrm{n}]$ is.

Calx viua; quyk-lym; viue chauz.

Cucurbita agrestis; col, stonel.

175 Carduus fullo[n]is; sauuonere; crowsope.

Cuscuta; id est dodere; [podagra lini].

Colubrina; dragaunce; edder-wort.

Corona regia, mellilotum, trifolium; mellilote; pre-leuyd gres.

Catapucia; spourge.

180 Copiosa; herbe copie.

Crocus affricanus, crocus ortensis; saffron de ort.

Custos ortorum, palma Christi.

Chimolea, terra sigillata.

Calculum, c[a]rdamomum.

185 Calcantum, attramentum, vitriolum.

Calcecumenon, es vstum, cachitheos.

$\mathrm{Ca}[\mathrm{me}]$ pitheos; medo-ratel.

Camphorata; aueroyne; sowperenewode.

p. 250 Cameactus, ebulus; | walwort; eble.

190 Camelee, quinque-folium, pentafilon; synkfoyl.

Camal $[\mathrm{e}] \mathbf{o n}[\mathbf{t}] \mathbf{a}$ is a pistel; i $d$ est remyng-worty $n$.

Cantarydes; buy, vlyen; grene wermes growyng in asschys.

Cancer marinus; a crabbe of pe see.

Cardo az[inin] us, cardo comunis; pystil.

195 Cardo benedicta, labrum Veneris; pow-pistil.

Catholica; laureola.

[Carica], ficus sicca; dreye figge.

Carei [pontici]; dauck.

Cardam[um]; toun[c]ressez.

200 Catha[ri] atici, rasura cor[nu] cenui; pe chaungyng of hertishorn.

Caride miristice, id est nucis mus[c]ate; note-mugys.

Cassia lignea, xilocassia.

172 cymynum] ymymim $M S$. | anis] auis $M S .175$ fullonis] fullouis $M S$. 176 podagra lini] padagralini $M S .184$ cardamomum] cordamomum MS. 187 camepitheos] canapitheos MS. 191 camaleonta] camalconca MS. 194 azininus] azunius MS. 197 carica] cariaca MS. 198 pontici] carei porcini MS. 199 cardamum] cardami MS. | touncressez] touneressez MS. 200 cathariatici] cathanatici MS. | cornu] cormi MS. 201 muscate] mustate MS. 
Cassia fistula is pe fruit of a tre, but cassia lignea is pe rynde of a fruyt.

205 Castorium is be ballok of a beste.

Cauda pulli; a poddok pype.

Cauda equina; foxes-gloue.

Cel[s]us, mora maior; mulberye.

[S]pica celtica, cestrum; betoyne.

210 Centaurea maior; centorie; Cristes-laddere.

Centonica, absynthium ponticum; se-wormod.

Centrum galli, gallitricum, gallici [cr]ista; centre galle.

Centineruia, plantago, arnoglossa, lingua agni; lambis-tunge.

Dactulus; a date.

215 Dactulus acetosus, oxif[eni]cia, finicon; tamarydes.

$\mathbf{D a}[\mathbf{u}]$ cus creticus, trencus; dent de leon.

Dragancia, colubrina, cerpentaria, cocodrilla, basilicus; neddrewort.

Dragancia argirofora, rapistrum; cherloke.

Dascripta, epatica; liuerewort.

220 [Daphnides]; pe tre of lorer.

[Daphneleon], oleum laurinum.

Dendro-libani, [libanotidos], anthos, ros marinus; rose-maryn.

Dens equi, iunccus, triangulatus; ciperus.

Demachian, m[iltro-]datum, sanguis draconis.

225 Diptann $u s$; dytandre.

p. $251 \operatorname{Dr}[\mathbf{i}] \mathbf{o p t}[\mathbf{e}] \mathbf{r i}[\mathbf{s}]$, pol|lipodium.

Dionisia, flos citoree; pe flour of ruddene.

Epatica, dascripta; coupere; liuerwort.

Eupatorium, ambrosia, lilifagus, saluia agrestis; hyndhale.

230 Edera terrestris; yuy.

El[io]tropia, solsequium, verucaria, cicorea, sponsa solis, mira solis; solcicle, [ruddene].

Elemp[ni] um, e[n]ula campana; horselne.

213 centineruia: [ce]ntumneruie added in the outer margin by a later hand. 222 anthos: uous added in the inner margin by a later hand.

208 celsus] cellus MS. 209 spica] cpica MS. 212 centrum] centum MS. crista] ista $M S .215$ oxifenicia] oxifoncia $M S .216$ daucus] dancus $M S$. 220 daphnides] dampnides $M S .221$ daphneleon] dampnileon $M S$. 222 libanotidos] liban tidos $M S .224$ miltro-datum] multus datum $M S$. 226 driopteris] droptori $M S . \quad 231$ eliotropia] eleutropia $M S . \quad 232$ ruddene] ruddenen MS. 233 elempnium] elempruum MS. | enula] emula MS. 
E1[a]cterium; pe ius of cucumbre, id est wilde papie.

235 Esbrium; pe flour of sawge.

E[tiop]is, b[ar]dana, lappa [in]uersa; clotere.

Ematithes, lapis giron, lapis sanguinaris.

Es vstum, calcecumenon; bras y-brent.

Endiuia, tro[x]ima; endyue.

240 Elacterides; pe iouys [catapus], id est spurge.

Euiscus, altea, malua viscus, bismalua; pe wise malwe.

Eleborus niger; clouetunge, blak elebre.

Eleborus albus, [scelerata]; whit elebere.

Epithimum; flour of tyme.

245 Eruca; skyrwhit.

Euforbium; euforbe; it is a gumme of a tre.

Eriscus; colophonie; resina, pix greca.

Faba; a bene.

Fabaria; lemyk; fauerole.

250 Farina ordei, alphita; flour of barlich.

Frag[a]ria; frasere; streberiewyse.

Frammulus; slepwort.

Fraxinus is a tre pe whic ys clepid an assch.

Fragrum, bruscus; bonwort.

255 Falgeria, filex, fengus; fern.

Febrifu $[g] a$, fel terre, centaurea, symphonica; feperfoye.

Fenigrecum; femigrek.

Fex; a dreste.

Fel; galle.

260 Feniculus; fenel, pe sed ys clepid maratrum.

Feniculus porcinus, peusedanum, amarisca; houndeffenel.

Ferula is an herbe of pe iouys is mad galbanum.

Fermentum; sow $r$ dow3.

Filago; harewort.

265 Filipendula, sisalidos; ston-wort.

p. 252 Fi[ni]|con indi, oxifen[i]cia; tamaryndi; dactulus acetosus.

Flosmus, tapsus barbastus, p[o]nfilion, herba linaria; moleyne.

256 centaurea: fel bre added in the outer margin by a later hand.

234 elacterium] electerium MS. 236 etiopis] elix-is $M S . \quad$ bardana] brandana $M S . \quad$ inuersa] euersa $M S .239$ troxima] troynna MS. 240 catapus] cateprus $M S$. 243 scelerata] adarasca $M S$. 251 fragaria] fragria MS. 256 febrifuga] febrifuca $M S . \quad 266$ finicon] frincon $M S . \quad$ | oxifenicia] oxifencia MS. 267 ponfilion] penfilion $M S$. 
Flos ciriacus; flour of malwes.

Flos roris marini, anthos; flour of rose-maryn.

270 Flos fraxini, alphur; flour of pe assch.

Flos mali granati, balaustia; flour of pomegarnet.

Flos plumbi; ceruse, blank plum; flos eris; verdegres.

Fumus terre; houndhore; fumitorie.

Fulfulabiat, piper nigrum; blak pepir

$275 \mathbf{F u}[\mathbf{l f}] \mathbf{u l e s b e t h}$, album piper, whit pepir.

Furfur, cantabrum; bren of whete.

Fructus iuniperi, amy fructus; pe froyt of iuniperi.

Fructus edere, carpo[c]issi, baccarum edere; bayes of yuy.

Frumentum; whete.

280 Galbanum is pe ious of an herbe.

Galla, pomu $m$ quercinum, sicidon, [alapsa].

Galanga; galingale.

Gallitricum, centrum galli, gallicristus.

G[a]riophilus; a clowe.

285 Gariophilata, sana-munda, pes leporis; auancia; auence.

Gariophilus agrestis, azabac[c]ara.

Gladiolus; gladiole.

Gra[men], a[gro]stis; cuchegras.

Grana solis, milium solis; gromyle.

290 Gagathes; geeth.

Gla $[\mathbf{u}]$ cia, celodonia; celydonye.

Genesta, mirica; geneste; brom.

Genestula is lich a brom wip a whit flour.

Geniculata, poligonia.

295 Genciana, allogallica.

Germandria; germandrie.

Gelena, coloquintida, cucurbita alexandrina.

Gersa; cerusa.

Gith, ag rimilla[t]um, melanc[i]um; cockel.

273 fumus: [ho]undhore added in the outer margin by a later hand. 299 cockel: [pa]lma Christi added in the outer margin by a later hand.

275 fulfulesbeth] fussulesbeth $M S$. 278 carpocissi] carpotissi MS. 281 alapsa] alapasa $M S . \quad 284$ gariophilus] griophilus $M S . \quad 286$ azabaccara] azabactara $M S . \quad 288$ gramen] granis $M S . \quad$ agrostis] amesistis $M S$. 291 glaucia] glancia $M S .299$ agrimillatum] agrimillacum $M S$. melancium] melancum MS. 
300 Gira solis, custos orti, mirac[u]la, pentadact[i]lus, palma Christi.

Glicida; pionia.

Giron, lapis ematithes.

Glicia, licoricia.

Glis, e[tiop]is, lappa, bardana, illafeas; clote.

305 [C]risolocanna, attriplex agrestis; arrage.

Gliconiun, pullegium regale, puliole rialus, $[\mathrm{ev}] \mathrm{ic}[\mathrm{t}] \mathrm{us}$, crocus; p. 253 saffe|ron.

Gi[r]on, lapis magnetis.

Gisastereos, bolus armoniacus, it is a red erthe pe whiche is foundyn in Armonye.

Glumen arabic whan it is wretyn sympleleche gumme (of gumme arabic it schal be understonde).

Juncus, [podagra lini], cuscuta idem est.

Hastula regia vel hasta regia; woderoue.

315 Hastula Sancti Christofori, pollicaria minor; scha[bb]ewort.

Herba benedicta, ci[c]uta, incubus; homeloc.

Herba paralisis, herba Sancti Petri; cowslop.

Herba Roberti, acus muscata, pes columbe.

Herba perf[o]rata, ypericon, herba Iohannis, scopa regia; Seynt Johanis wort.

Herba Walteri; herbe Walter.

Herba acetosa, acedula, [o]xilapacium, rume[x]; surel; sourdocke.

Herba lunaris, tapsus barbastus, flosm $u s$, ponfilion, molena; moleyn.

Herba cruciata; herbe croyse.

325 Herba medee; mede-wort.

Herba fullonum, saponaria; $\mathrm{b}[\mathrm{o}] \mathrm{ri} 3_{\text {th }}$, crowesope.

Hermodact $[\mathbf{i}] 1[\mathbf{u}] \mathbf{s}$ is an herbe pe whiche pe rote is co[uen]able to medicines.

Herba Sancti Pauli; spergula.

303 glicia: [pi]oniae added in the outer margin by a later hand. 318 acus: pes colum[be] added in the outer margin by a later hand.

300 miracula] miracla $M S$. | pentadactilus] pentadactulus MS. 304 etiopis] elixis MS. 305 crisolocanna] grisolocanna MS. 306 evictus] wicus $M S$. 308 giron] gichon $M S$. 313 podagra lini] podagralini $M S$. 315 schabbewort] schawewort MS. 316 cicuta] cituta $M S .319$ perforata] perferata $M S$. 322 oxilapacium] exilapacium $M S$. $\mid$ rumex] rumei $M S$. 326 borizth] berizth MS. 327 hermodactilus] hermodactules. | couenable] couable MS. 
330 Hirenia \& huenea, hipposeta, cauda equina, cauda caballina. It is a row herbe wip pe whiche men pulschyn cambes, bowes and cuppes.

Hippia maior and minor, Hippia maior, morsus gal[1]ine; chekenemete. Hippia minor; selfhele.

335 Jacea nigra; matefeloun wip a red flour.

Iacea alba; matefeloun wip a purple flour.

Iarus, pes vituli, barba Aaron; zekesters.

Ilapheas, bardana, lappa; clotre.

Insana, ius-quiamus; hennebane.

340 I[nv]iola alba; whit violet.

Incubus, cicuta, herba benedicta; homelok.

p. 254 Incuba, sponsa solis, sicorea, mira solis, verucaria; | rudune.

Indi dactilis, acetosus, oxifen[i]cia; tamaryndis.

Ipericon, herba perforata, herba Johanis, scopa regia; Seynt Iohanis wort.

Ipia maior, morsus galline; chekenewed.

Ipia minor; [wey]-wort, eyze-wort.

Ipoquistidos is a superfluyte of growyng on pe erthe pe whic beryth rosa canina.

350 Iris hap purpil flour.

Ireos hap a whit flour. An-oper is clepid gladiole and he hap a zelw flour lik saffron, [an-oper] is cleped spatula fetida and he hath no flour.

Isopus; jsope.

355 Iouis barba; sel[f]grene.

Iuniperus, iuniper.

Eringi; secacul; [y] ringes.

Ision; centorie.

Katariacum, rasura cornu cerui; pe changyng of hertis-hornes.

$360 \mathbf{K a}[\mathbf{c}]$ abre; vernych.

Kalendula, solsequium; Seynte Marie rude.

Kufordaphin, cynamomum; canel.

Labrusca.

334 hippia: selfhele added in the outer margin by a later hand. 361 kalendula: [Se]ynt Mary gold added in the outer margin by a later hand.

333 galline] galine $M S . \quad 340$ inviola] ivuiola $M S .343$ oxifenicia] oxifencia MS. 344 scopa] Iscopa MS, I then erased. 347 wey-wort] vlex-wort MS. 352 an-oper] Ian oper $M S .355$ selfgrene] selgrene $M S .357$ yringes] pringes MS. 
Labrum Veneris, labium, cardus benedictus; pe sed is clepid Seynte Marie sed.

Lactuca domestica; letuse.

Lapdanum is a gumme.

Lappa, b[a]rdana, glis, e[tiop]is; gletoner, clotre.

Lappac[i] um rotundum id est [meche docke]; rumex, parella.

370 Lappacium acutum; smal docke.

Lapis lazuli is a ston of which asure is mad of.

Lapis armenicus and lapis calaminaris bope gon to medicines.

Lapis pirrites; flintes.

Lapis magnetis; githon.

375 Loodestone, lanceo-lata, plantago minor, quinquenenuia; ribwort; launceole.

Lapis linxis comep of pe vrine of a wolf pissid on an hil and muntayn y-cruddede; [1]apis agapis.

Lapis iudaicus.

$380 \quad[\mathbf{L}] \mathbf{a}[\mathbf{c t}] \operatorname{arid}[\mathbf{e}] \mathbf{s}$, cathapucia, anabulla.

p. 255 Laureola, lapidon, alipiados; pe sed per-of is cocon|[i]dium.

Laurencia, bugla.

[L]auendula; lauendre.

Laurus is a lorere tre.

385 Lentiscus is a tre pe which swetyp out oyle.

Lempnias; orpement; arsenik.

Le [u]isticum; louache; louestiche.

Lenticula aquatica vel lentigo; e[n]edemete; [lentil d'ewe].

Lectorica, nux coruli; filberdis; auellana; walnote; nux parua, nux pontica.

[Lepides] calcis, batitura eris, squ[a]ma eris; schalis of bras.

Leporina, pria[pi]scus, satirion.

Lepus siluestris; an hare.

[Leucopiper], piper album.

395 Libanum, olibanum, thus.

Libanotidos; ros-marine.

364 be: [S]eynt Marie [s]ede added in the outer margin by a later hand.

368 bardana] bordana $M S$. | etiopis] elexis MS. 369 lappacium $^{1}$ ] lappacum MS. [ meche docke] mechedocke MS. 378 lapis $\left.^{1}\right]$ apis MS. 380 lactarides] attaridos MS. 381 coconidium] cocondium MS. 383 lauendula] avendula MS. 387 leuisticum] lemisticum MS. 388 enedemete] euedemete MS. lentil d'ewe] lentildewe $M S . \quad 391$ lepides] lempidos $M S$. | squama] squma MS. 392 priapiscus] prianuscus MS. 394 leucopiper] leuco MS. 
Ligustrum; [wilde lilye].

Lilium; lilye.

Lilifagus, sal[u]ia agrestis, ambrosia, eupatorium; hyndehale.

400 Licosperma; seme $n$ saxifrage.

Linilion, olium lini; linsed.

$\mathbf{L i}[\mathbf{t}]$ hodemonis is a blak ston whic comep out of Ynde, cha[u]yd wip frotyng it drawip to hym straw; id est geet.

Licoricia; licoris.

405 Limphea aquatica, menphia, lilium aquaticum; nenufare, water of lilye.

Li[n]ochites, mercur[i]al[i]s.

Lingua canis; hundestunge.

Lingua ceruina, scolopendria; hertistunge.

410 Lingua bouina, buglossa; oxetunge.

Lingua ag[ni]na, arnoglossa, plantago, centinenuia.

Lingua canis; scikewort.

Lingua hircina, plantago minor, 1[a]nceolata.

Li[po]tomia, malf[actio], sincopis; [swonynge].

415 Lunarie purgep be colre pe which berith a whit flour.

Linoyse, spinache be which berip an ynde blew flour.

Longum piper; macro-piper.

Lollium, nigellam, agrimila[t]um, gith, melanc[i]um; id est cokkel.

Lupines, faba egypcia, mar[s]ilium; lupines.

420 Maba[t] ematicon; pe ious of wilde caul dreied.

p. 256 Maces, folium.

Macedonia; elizan $n$ dre seed.

Magnetes; 1[o]odeston.

Maculata, trifolium; pre-leuyd.

425 Maiorana, amaricus, amaricon, cimbrium, sansucus, persa; mugwort.

Malum macianum, malum macidum, pomum siluestre, wode-crabbe.

Malum punicum, malum granatum; pome garnet.

Mala coctana, mala citonia; coynzes.

412 canis: centumnueruie added in the outer margin by a later hand.

397 wilde lilye] wildelilye $M S . \quad 399$ saluia] salnia $M S . \quad 402$ lithodemonis] lichodemonis MS. | chauyd] chanyd MS. 407 linochites] limochites MS. mercurialis] mercurealus $M S .411$ agnina] aginina $M S .413$ lanceolata] lonceolata MS. 414 lipotomia] limpatomia MS. | malfactio] malfiton MS. swonynge] swenyngen $M S$. melancium] melancrum $M S$.

418 agrimilatum] agrimilacum $M S$. 19 marsilium] marfilium $M S$ 420 mabatematicon] mabafematicon MS. 423 loodeston] leodeston MS. 
Melan[o]piper, piper nigrum.

430 Malum terre, ciclamen, ca[s]samus, panis porcin $u s$; erthe-notes.

Maluaviscus, bismalua, a[l]thea, euiscus, ebiscus.

Matricaria, arthemesia; mugworth.

Matrisilua, caprifolium, oculus lucij, volubilis maior, caprisicus.

Macro-piper, longum piper.

435 Marsililium, faba lupina, faba egypciaca; lupynes.

Masmatrum, peucedanum, feniculus agrestis; hundesfenel.

Maratrum; fenel-seed.

Marrubium; hor-ho[un]d.

[Manna, ros] celestis; dew of heuene.

440 Mandragora; mandrake.

Malagma is mollificatyf en-plastre for betyng materie.

$\mathbf{M}[a]$ lasticum is a mollificatyf makyng materie.

Melaticum; dissoluinge.

Melandrea; berewort.

445 Mellicratum; mede mep.

Mel rosatum; mel roset.

Mellilotum, trifolium, maculata, corona regia, mellago, putella.

Melissa, ci $[\mathrm{t}]$ raria, herba pigmentaria, ci[t]rago.

Melones bup mundificatyf.

450 Melanc[i] um, gith, agrimula[t]um, nigella; cokel.

Menta anana; fischment.

Menta aquatica, mentastrum, sizimbrium; horsment.

Mentula episcopi; medeflour and hit hap a zelw flour and brode leuys, and it growip in pe water.

455 Mentula sacerdotis; circoure.

Mercurialis, linochites, par[t]eno[t]hidos, libleb.

Meu is an herbe lich hundesfenel, but it is more croked.

Menelaca; goldys, bothun.

Mesbili; nesfles; meeles.

460 Miltrodatum, domachian, sanguis draconis.

p. 257 Miconium, nichocodium, [papa]uer | album; whit papie.

Mill[e]folium, venter apis, supersilium Veneris; zarwe.

429 melanopiper] melanepiper MS. 430 cassamus] calsamus MS. 431 althea] athea $M S . \quad 438$ hor-hound] hor honud $M S . \quad 439$ manna ros] mannaros MS. 442 malasticum] molasticum MS. 448 citraria] cicraria MS. | citrago] cicrago $M S$. 450 melancium] melancum $M S$. agrimulatum] agrimulacum MS. 456 partenothidos] parcenochidos $M S$. 461 papauer ] parpuer $M S$. 462 millefolium] millofolium $M S$. 
Milleum solis, granum solis, cauda porcina; id est gromolye.

Mirtus est frutex and frutes per-of bep clepid mirtellis, hurtene.

465 Mirta est frutex and it growep in morys; id est gawl.

Mira solis, cicorea; id est ruddene.

Mirabolanes bep frutes and per bep fyve diuers maner per-of: kebul, yndorum, emblicorum, citrinorum and belricorum.

Mirra; pe gumme of a tre.

470 Mirica; genesta; brom; genest.

Mora; blake beries.

Mora Celsi; molle-beries.

Morella, strignum, solatrum, herba Sancte Marie; morell.

Morsus dyaboli; recoupe.

475 Morsus galline, ypia; chekenemete.

Molena, tapsus barbastus; moleyn.

Mummia is a spice pe whiche is foundyn in pe graues of dede men and it is pe ious of pe body j-runne to-gedere a-noyntyd wyp bawme.

480 Muscata, hasta regia; woderoue.

Muscus is pe humitide of a beste.

Mulsum is mad of viij parties of water and pe ix parti of hony and $j$-sodyn to be consumcyoun of pe pridde partie.

Mus on Englisch is a mous.

485 Narcha is a fisch pe which whan he is drawen wip hook and lyne and $3^{\text {if }}$ pe fischere putte his hand vp on hym it makep pe hondyn vnfredable and al pe body after, but it be pe rapere takyn a-wey and per-of men makyn medycyn to make men to slepyn.

490 Nastare is a suppositorie or a passarie.

Nasale is an instrument to castyn in medicynes be pe nosetherles.

Nasturcium aquaticum; watercresses.

Nasturcium ortolanum; tuncresse.

495 Nasturcium porcinum, sanguinaria, bursa pastoris; schepherdis powch. |

p. 258 Nasturcium agrestis; wildecresse.

[Nardostachium]; spicanardi; spikenard.

Napta, petroleon; oyle of til stones; oleum benedictum.

500 Napium, semen sinapis; mustard seed.

Nardilium; oyle of spikenard.

498 nardostachium] nardostanchium MS. 
$\mathrm{Ne}[\mathrm{n}]$ uphar, [nim]phea and pe rote is clepid caballina.

Nimphea, lilium aquaticum; id est ne[nu]phar, water of lilie.

Nepta; be lasse calamynte; [cattes ment].

505 Nigella, agrimula[t] um, gith, mellanc[i] $u m$; kockel.

Nitrum is a veyne of pe erthe or a kynde of salt.

Nux maior; a walnote.

Nux muscata, nux miristica; notemuges.

Nux vomica; chesteynes.

510 Nux pontica, nux parua, auellana, lectorica, nux coruli; notes of haselis.

Nucleus; pynes.

Obtarticum, sternutarium is pyng to make men vnese.

Obtalmicum, lapis calaminaris; calamine.

515 Oculus Christi is good for eyzen.

Oculus bouis; id est bothun.

Oculus populi pe beries of pe popeler.

Offei, gumme edere; gumme of yuy; opo[c]isci.

Olium lenci.

520 Olium oliue; comune oyle.

Olium lini; le[n]elion; oyle of linsed.

Olium lapidum, olium petrolium, olium benedictum.

Omentum, abd[o]men, sumen, auxungia, pinguedo; fatnesse, gres and whan it is put symplelich be hym-self swynes gres it schal ben vnderstonde.

Olibanum, libanum, thus; frankensens.

Olixatrum, alexandrum, petrosilium; alisaundre.

Opium [thebaycum] is pe ious of whit papie.

Opium jus-quiami, opium miconis; pe ious of hennebane. Whan opium is put symplely by hym-self of opium thebaycum it schal be vnderstonde.

Op[o]ponak is pe gumme or pe ious of an herbe.

p. 259 Opepirus is pe clene bred fro pe bren of | payndemayn.

533 payndemayn: payndemayn Opbalsamum is pe ious of bawme Orobus milium mouse pece Ordeum barlich Origanum $\mathrm{O}$ added in the outer margin by a later hand.

502 nenuphar] nemuphar $M S$. | nimphea $\left.{ }^{1}\right]$ menphea $M S . \quad 503$ nenuphar] neunphar MS. 504 cattes ment] castesment MS. 505 agrimulatum] agrimulacum MS. | mellancium] mellancum MS. 518 opocisci] opotisci MS. 521 lenelion] leuelion $M S$. 523 abdomen] abdumen $M S$. 528 thebaycum] theobaycum MS. 532 opoponak] opeponak MS. 
Opobalsamum is pe ious of bawme.

535 Orobus, milium; [mouse-pece].

Ordeum; barlich.

Origanum; orgon.

Orton, verbena; verueyne.

Os spie; mensschelle.

540 Os de corde cerui; pe bon of an hert.

Os-munda; [e]uerferne on pe ook, polli-podie.

Oxifen[i]cia, finicio, dactulus acetosus; tamaryndes.

Oxi; vinegre.

Oxiren; [strong wyn].

545 Oxilappacium, acedula, rume[x], herba acetosa; surell; sourdokke.

Oxicantum, berberes.

Ozimum, seme $n$ basiliconis, ozimum.

Oygia, acantu $m$, vrtica; pe seed of netles.

Papauer album, codion; [blanc susement]; whit popie.

550 Papauer nigrum; no[ire] susement; blak popie.

Palma Christi, cauda porcina.

Pampinus, folium vitis; pe leef of vine.

Pamphiligos, flosmus, tapsus barbastus; moleyn.

Panis cuculi; wodesoure, allehiia.

555 Panis porcinus, ciclamen, malum terre; erpenote.

Plantago; plantayne; weybrede.

Pla-tociminum, sile[r] montanum; surm[o]ntayne.

Passula, passulus, v[u]a passa; reysynges.

Pastinaca, baucia; paster-nep.

560 Passerina, lingua corrigiola, geniculata, pros[er]pina, poligonum; swynes-gres.

Prassum, porrum; lek.

$\mathbf{P}[\mathbf{r}]$ assium viride; verdegres.

prassium; horoune; marrubium album; moriell.

$565 \mathbf{P}[\mathbf{a}]$ ritoria; paritorie.

Par[di]ciados, herba vitriola, herba muralis; paritarie; stonhoue.

Pet[a]la, cortex citri.

541 euerferne] ouerferne $M S . \quad 542$ oxifenicia] oxifencia $M S . \quad 544$ strong wyn] strongwyn $M S . \quad 545$ rumex] rumei $M S . \quad 549$ blanc susement] blancsusement MS. $\quad 550$ noire] nom MS. 557 siler] silei MS. surmontayne] surmentayne $M S . \quad 558$ vua] vna $M S . \quad 560$ proserpina] prospina $M S . \quad 563$ prassium $\left.^{1}\right]$ passium $M S . \quad 565$ paritoria] peritoria $M S$. 566 pardiciados] parciados $M S$. 567 petala] petula $M S$. 
Pentafilon, quinque-folium, camolee; v-le[u]ede gras.

Penttadact[u]lus, palma Christi; swynes-tayles.

570 Petrosillium [macedonicum]; Alisandre-sed.

Petroleum, oleum de pet $r a$, oleu $m$ benedictum.

Pes leporis, auancia, sanamunda, garofilata; auence.

$\mathbf{P}[\mathbf{e}]$ ric[li]menon, matrisilua, caprifolium, capriscus, oculus lucij, |

p. 260 volubilis maior; wodebynde.

575 Peristereon, columbarea, venuena.

$\operatorname{Per}[c]$ icaria minor, pessicaria, cullirabies; cul-rage.

Percicaria maior; droppewort.

Piganus, pulmonaria, palla marina vel pella; bellewort.

Persa, maiorana, cimbrium, sancsucus, amaracus; muchewort.

580 Pepones, melones.

Pes nisi, pulmonaria, politricum; sperhawkfot.

Pes vituli, jarus, barba Aaron; zekesters.

Pes corui, apium emeroydarum; crowefot.

Pes pulli, andrago.

585 Pes columbe hap leuis departid in vij and euery party hap to departynges or v.

Pes [anticipitris]; columbine pe same.

Peucedanum, feniculus porcinus; hundesfenel.

Penidie; penides.

590 Picula, pix liquida; pich.

Pistacee bep fruites lich pines.

Piga[no]n; wilde rue.

Pigula, lingua a $[u]$ is; stichewort; pigle.

Piper album, ful[ful]abiat.

595 Piper longum, melanopiper.

Piper nigrum, ful[f]ules-beth macropiper.

Pilocella, chaunet; mousere.

Pigmentaria, melissa, citrago; mellilote.

[Psidia], cortex mali granati.

600 Psillium is pe sed of an herbe.

Piretrum; peletre of spayne.

Pollium marinum, pollegium.

568 v-leuede] v lenede $M S . \quad 569$ penttadactulus] penttadactalus $M S$. 570 macedonicum] mascedonicum MS. 573 periclimenon] parichemenon MS. $\quad 576$ percicaria ${ }^{1}$ ] perticaria $M S . \quad 587$ anticipitris] ancipatris $M S$. 592 piganon] pigamen $M S . \quad 593$ auis] anis $M S . \quad 594$ fulfulabiat] fulsinabiat MS. 596 fulfules-beth] fulsules beth MS. 599 psidia] picidia MS. 
Pollium montanum, poloes; puliole mountayne.

Propoleos; whit wax.

605 Policaria is an herbe and pe sed per-of is clepid pusillium.

Pollicaria minor; skabbewort.

Pollipodium; fe[u]gerole; ouer-fern growyng in an ok.

[Podagra lini], cuscuta; doder.

Portulaca, [albagla, adragmis], pes pulli, porcellan $a$; porcellane.

610 Pomum quercinum, galla, scicidon, alapsa; ok appel.

Pomum citrinum; pome citre.

Pomfilion, flosmus, tapsus barbastus; moleyn.

Pullegium, gliconium, golena; puliole.

Pullegium Martis, diptanus, dittaundre.

615 Plumbum; leed.

Pruna; a pl[u]me.

Prunella; a sloo. $\mid$

p. 261 Quercus; an ok.

Quercula maior, camapitheos; moderatell.

620 Quercula minor, camedreos; germandrie.

Qvinque-neruia, plantago minor, lanceolata; ribbewort.

Quinque-folium, pentafilon, camoleon; synk-foyl; bat is to seynne fyue-leuyd gras.

Radix whan it is put symplich of raphanus (id est radix) it is schal be vnderstonde.

Rapistrum, dragancia; cherl-ok.

[Rasca lini], podagra lini, cuscuta; dodere.

Regina, aquileia, angelica; remet; moderwort, medewort.

Resta bouis; cammok.

630 Resina, rosyn.

Rami cedri, lignum imperij; ecoree, recopee; morsus demonis; forbitene.

Risi; rys.

Rubarbe is pe rote of pe lasse geneste.

635 Reuponticus, rosa rubia; red rose.

Rosa alba; whit rose.

[Rodoxiron], olium rosarum; oyle of rosyn.

Rodost[ag]ma, aqua rosarum; water of rosyn.

607 feugerole] fengerole $M S$. 608 podagra lini] podagralini MS. $\mathbf{6 0 9}$ albagla adragmis] abaga adriagnis $M S . \quad 616$ plume] plime $M S . \quad 627$ rasca lini] rascalini MS. 637 rodoxiron] rodoxioron $M S .638$ rodostagma] rodostoma MS. 
Ros marinus, anthos, dendeolibanus.

640 Ros ciriacus; pe flour of malwes.

Rostrum porcinum; sow3 bystel.

Robelia; pe com[o]un pes.

Rubia maior; warencia; madu[r].

Rubia minor; heirif, clipen.

645 Rubus, rumex, tribulus; bremell, whete whil pat it berip manye brembelis, but whan rubus is symplech put, as pe brembil pat berip blake beryes it schal be vnderstonde.

Sagapinum, serapinum, sa[n]sucus, amaricon, amaricus; maiorane; muchelwort.

650 Sambucus, actis; elderene.

Sanguis draconis, miltrodatum, dema[c]hian; sank draconum.

Salix; a welw3-tre.

Saliunca, spica celtica.

Sal[u]ia, salgia; sawge.

655 Sal; salt. Per bep manye maner of saltes: sal comun, sal armoniak,

p. 262 sal gumme, sal cap[a]docium, sal [tragecicum], sal pensum, | sal sacerdotale, sal macelli, sal catarticum, sal [nitrum], sal alcali, sal tartari.

Saundres, per bep pre spices of hem: white, rede and citrine.

660 Sanguinaria, bursa pastoris.

Sanicla; sanicle; wodemerch.

Sandix; wood.

Sana-munda, pes leporis, garophilata; auence.

Sandonicum; [se wormood].

665 Sandar[a]ca; vernych.

Sapa, id est nouu $m$ vinum; in tyme of vines must.

Saponaria; sauener; crowe-sope, notebrun.

Satirion, $\mathrm{p}$ [riap]iscus, leporina.

Satureia, tymbra; sauereyne.

670 Sar[c]oc[o]lla; pe gumme of a tre.

Sauina, bracteos, cariola siluestris; wilde letuse.

Staffi-sagria; an herbe pur netes and les.

Scamonea, diagredium.

642 comoun] comeun $M S .643$ madur] madu3 MS. 648 sansucus] sausucus MS. $\quad 651$ demachian] demathian MS. 654 saluia] salnia MS. 656 capadocium] capodocium MS. | tragecicum] tracencium MS. 657 nitrum] mirum $M S$. 664 se wormood] sewormood $M S$. 665 sandaraca] sandarica MS. 668 priapiscus] paniscus MS. 670 sarcocolla] sartocalla MS. 
Saxifragia, a[sin]alici.

675 Scatuncellus, vmb[i]licus Veneris, cimbalarium; peny-wort.

$\mathrm{Sp}[\mathrm{a}]$ ragus, alion.

Spatula fetida; gladene pe whic berip no flour.

Se[t]acul, yrynge; seholme.

Sepha, ysopus.

$680 \mathrm{~S}[\mathrm{c}]$ elerata, aquilaria, apiu $m$ risus, $\mathrm{b}[\mathrm{a}] \mathrm{tracion}$.

Selinum, semen apij; [merch sed].

Semen vrtice, acantu $m$, basara, achiron, anagallidos; nettel sed.

Semper-viua, herba Aaron; self-grene.

Semen bulli; semen grosse cepe; oynonet.

685 Semen rose, antera; pe seed of roses.

Semen lini; lynsed.

Semen saxifrage, acinalici, licospermatis.

Semen rapistri, armon[i] aca; tuncresse-seed.

Senacio, nasturticum aquaticum; id est water-cresses.

690 Sen $[\mathbf{e}]$ cio, sonicium; groundiswilie.

Sene; id est a laxatif.

Saluatelle bep ij veynes in pe hondes be-twen pe litil fynger and pe fynger next hym and two on pe to fet in pe same place.

Stercus caprinum; pe dunge of a goot.

695 Stercus vaccinum; cowes dunge.

p. 263 Stercus ouium; | schepis dunge.

Stercus columbinum; [colueres dunge].

Stercus anatis; mathelardes dunge.

Stercus irundinis; swalwes dunge.

700 Stercus muris; mouses dunge.

Serpillum; peletre.

Serpentaria, dragancia, colubrina, cocodrilla; nedderwort.

Scicida, brionia; [wilde nep].

Simphonica, caniculata, iusquiami; hennebane.

705 Simphitum, anagallis, anagallicum, consolida maior; co[m] firie.

Sinapis; [mustard sed].

Sinonum, petrocilium agreste; wilde perselised.

674 asinalici] almalici $M S . \quad 675$ vmbilicus] vmbelicus $M S . \quad 676$ sparagus] speragus $M S . \quad 678$ setacul] secacul MS. $\quad 680$ scelerata] selerata MS. batracion] botracion $M S . \quad 681$ merch sed] merchsed $M S .690$ senecio] senicio MS. 697 colueres dunge] colueresdunge MS. 703 wilde nep] wildenep MS. 705 comfirie] counfirie MS. 706 mustard sed] mustardsed MS. 
Sigillum Sancte Marie; Seynte Marie flour.

Squilla, cepa marina.

710 Squilles; se oynonys.

Squina[n]tum, esca camellorum; chamayles mete.

Sisimbrium, balsamita; bawme.

Strignum, solatrum; morell.

Spica celtica; spykenard.

715 Sicomorus, ficus fatua; it is good for hem pat bep loue-sek.

Spinachia; spinache.

Stincti, stigni; pei bep fysches estenche, water nettes.

Sister, anetu $m$ dulce.

$\mathrm{S}[\mathrm{mirn}]$ is; betoyne.

720 Sili[n] um, semen apij.

Siseleos, siler montanum; surmountayne.

Sicla, beta, bleta, betis.

Siriaca, molachia, malva; malwes.

Stipteria, alumen, zuccarinum, igia, storax liquida.

725 Sinabrium, minium, vzifur; vermeloon.

Spodium, os elephantis; pe bon of an olephaunt y-brent or yuori brent.

Sponsa solis, solsequium, [c]icorea, incuba, verucaria, el[iotro]pia; golde flour.

730 Scordion, allium agreste; hare-belle.

$\mathrm{S}[\mathrm{c}]$ olopendria, lingua ceruina; hertistonge.

Sc[o]pa regia, herba perforata, herba Johannis, centum foramina, ypericon, fuga demonum; Seynt Iohannis wort.

$\mathbf{S}[\mathbf{c}]$ rofularia, s[te] robilea, pinea.

735 Sulphur viuum, cibapirum, tibapirum.

Sumach, anagodan.

Sturcium, caulilus agrestis, cauliculus ag[r]estis, brasica; smal caul.

Succus prunellarum, acacia; pe ious of wilde slon. |

p. 264 Spuma marina; pomys.

740 Succus frumenti, amidum, amilum; amydoyne.

Supercillium Veneris, venter apis, mille-folium; 3 arwe.

Tamariscus; [t]amaryndes; oxifen[i]cia, dactulus acetosus.

711 squinantum] squinatum $M S . \quad 719$ smirnis] sirinis $M S . \quad 720$ silinum] silium $M S . \quad 728$ cicorea] ticorea $M S$. | eliotropia] elentrepia $M S$. 731 scolopendria] stolopendria $M S$. 732 scopa] scepa MS. 734 scrofularia] strofularia $M S . \quad$ sterobilea] scrobilea MS. 737 agrestis $\left.^{2}\right]$ agestis $M S$. 742 tamaryndes] amaryndes $M S$. | oxifenicia] oxifencia $M S$. 
Tapsus barbastus, flosmus, herba lunaris; moleyn.

Tartarum, petra vini; argoil.

745 Tarascon; scariole.

Tanacetum, terbentina; terbentyne.

Terra hispanica, chimole[a].

Terra sigillata, terra sarazenica, argentaria.

Terra figuli, argilla, creta; crokeres cley.

750 Tumbra, timbria, satureia; saturegia; sauereyne.

Tibapirum, cibapirum, sulph $u r$ viuum.

Triticum; whete.

Trisogonus, camedreos, germandria, quercula minor.

Thim[o]n; tyme.

755 Tyt[i]mallis; per bep manye spices of hem: walwort, esula, anabulla, catapucia, laureola and opere.

Tisan $a$; it is pe decoccioun of water and barlych.

Tribulus, rumex, rubus; rounce; red brere.

$\mathbf{T}[\mathbf{r}]$ oxima; endyue or scariole.

760 Turiones, summitates vitis; pe croppes of vines.

Thus, olibanum; frankensens.

Ualeriana, fu, amantilla, potentilla, maturella, zodoarium agreste; valerian.

Uva acerba; a sauour grape.

765 Uua passa; reysons.

Uaccinium, morum nigrum; mirtilles, hurtene.

Uenter apis, supersilium Veneris, mille-folium; zarwe.

Uva lupina, strignum, morell.

Uerucaria, cicorea, incuba; ruddes.

770 Uermes terrestres, lumbrici terrestres; anglet-wacches, wermes of pe eerpe.

Uermicularis, crassula minor; stoncrope.

Uervena, $\mathrm{p}[\mathrm{er}]$ isteron, herba Veneris.

Uernyx, bernix, [classa].

775 Vespertilio; reremous.

Uigellum, pobel.

p. 265 Uirga pastoris, | ozoragi; wilde tesil.

Usifur, minium, vermiculum, cinabrium; vermeloun.

Uiola, violaria; violet.

747 chimolea] chimoled MS. 754 thimon] thimen MS. 755 tytimallis] tytumallis MS. 759 troxima] toxima MS. 773 peristeron] pisteron MS. 774 classa] classia $M S$. 
780 Uiola alba, jw, rathehele, vnctuosa.

Uitis agrestis, labrusca, viticella.

Uitriola, herba muralis, paritoria, per[di]ciados; paritorie.

Uitriolum, dragancium, calcantum; arrement.

Uiscus quercinus; [wi de] keyne, mystel of pe ok.

$785 \mathrm{Vmb}$ [i]licus Veneris, cotilidon, cymbalaria; peny-wort.

Vul[g]ago, baccaria, garofilus agrestis.

Volubilis maior, edera arborea, corigiola, caprifolium.

Volubilis minor, edera terrestris; heyhoue.

Vrtica mortua, archangelica, barba angeli; blynde nettel.

790 Vrtica greca; ruge vrtie; red nettel.

Vngula caballina is pe rote of water lilye or ellis pe rote of memphe.

Xil[o]cassia, cassia lignia, cassia rubia \& sicca, [x]ilobalsamum, lignu $m$ balsami; pe fruyt per-of is clepid carpobalsamum.

[Psylli]um, [z]archaton.

795 Ypericon, perforata, centum foramina, herba Johannis; Seynt Johannis wort.

Ytea, salix; welw.

Yriana, labrusca, vitis agrestis.

Ypia maior, morsus galline; chikenemete.

800 Yris, purpureum flore gerith.

Yreos, albu $m$ gladiolus, croceum set spatula fetida [n]ullum.

Yris hap a purpil flour.

Yreos hap a whit flour.

Yposilea, fabaria; lemyk; fauerole.

805 Ypoquistidos is a round bal growyng a-bowte a brembill.

Ydromel is a water wip hony y-sode.

Ygia, ozimium; nettel seed.

Yposarcan ys a dropesye of malencoly.

Ypomaratrum; wilde fenel.

$810 \mathbf{Z a}[\mathbf{r}]$ chaton, [psyll]ium.

Zedoarium; zedewale.

[Z]ima, fermentum; leueyne.

p. 266 Zinnia is a | postem of fleume.

793 fruyt: Nota $\mathrm{b}[$ ene $]$ added in the outer margin by a later hand.

782 perdiciados] perciados $M S . \quad 784$ wi de] wilde $M S . \quad 785$ vmbilicus] vmbelicus $M S .786$ vulgago] vuldago $M S .792$ xilocassia] xilacassia $M S$. xilobalsamum] ilobalsamum MS. 794 psyllium] ypsillum $M S$. | zarchaton] archaton MS. 801 nullum] mullum MS. 810 zarchaton] zachaton MS. psyllium] ypsillium MS. 812 zima] ima $M S$. 
Zinzelcon, pentafilon; v-leued gras.

815 Zipule, crispule.

Zinziber; gynger.

Zucc[a]ra; zugre.

Zirbus; grece, fattnesse.

Zirungen; borage.

820 Zizannia, nigella, gith, lollium; cockell.

817 zuccara] zuccura MS. 
